\title{
Predicting effective factors on eating behaviors in the prevention of cardiovascular disease based on the PRECEDE model
}

\author{
Samera Radmerikhi ${ }^{1}$, Seyed Vahid Ahmady Tabatabaei ${ }^{2}$, Yunes Jahani ${ }^{3}$, Mohabbat Mohseni ${ }^{4}$
}

\begin{abstract}
${ }^{1}$ MSc. of Health Education, Social Determinants of Health Research Center, Institute for Futures Studies in Health, Kerman University of Medical Sciences, Kerman, Iran

${ }^{2}$ M.D-MPH-Ph.D., Assistant Professor, Social Determinants of Health Research Center, Institute for Futures Studies in Health, Kerman University of Medical Sciences, Kerman, Iran

${ }^{3}$ Ph.D. of Biostatistics, Assistant Professor, Modeling in Health Research Center, Institute for Futures Studies in Health, Kerman University of Medical Sciences, Kerman, Iran

${ }^{4} \mathrm{Ph} . \mathrm{D}$. of Public Health and Health Promotion, Assistant Professor, Social Determinants of Health Research Center, Institute for Futures Studies in Health, Kerman University of Medical Sciences, Kerman, Iran
\end{abstract}

Type of article: Original

\begin{abstract}
Background and aim: Changes in eating behavior can reduce the risk of developing cardiovascular disease. The aim of this study was to predict the effective factors of eating behaviors in the prevention of cardiovascular disease using the PRECEDE model.

Methods: This cross-sectional study was performed on 400 subjects aged from 20 to 60 years old in Kerman, Iran in 2016. The participants were selected using a multistage random sampling method. A self-administered questionnaire including questions regarding demographic characteristics, eating behavior, and PRECEDE model constructs were completed by the participants. Data were analyzed using SPSS 22 and STATA 12. For data analysis, Spearman correlation coefficient, univariate and multiple median regression were applied. The predictive power of the model constructs was determined by analysis of artificial neural networks.

Results: Among participants, the score of knowledge was high $(84.15 \pm 10.7)$, and the scores of perceived selfefficacy (59.1 \pm 16.57$)$, reinforcing factors $(60.66 \pm 14.01)$, enabling factors $(56.5 \pm 12.91)$, and eating behavior (62.1 \pm 14.7$)$ were intermediate, and the score of attitude was low (47.84 \pm 7.67$)$. Attitude, self-perceived efficacy, enabling factors, and knowledge predicted $32 \%, 30 \%, 26 \%$, and $0.93 \%$ of participants' eating behavior respectively. The relationship between all variables and eating behavior was positive and significant $(\mathrm{p}<0.0001)$. Perceived self-efficacy had the most, and reinforcing factors the least correlation with eating behavior.

Conclusion: According to the results of this study, self-efficacy, attitude, and enabling factors were the main predicting factors for eating behaviors; therefore, to prevent cardiovascular disease and enhance healthy eating behavior, it is recommended to change attitude, and enhance self-efficacy and enabling factors in the community.
\end{abstract}

Keywords: Eating behaviors, Cardiovascular disease, Predisposing factors, Reinforcing factors, Enabling factors

\section{Introduction}

Cardiovascular disease (CVD) is one of the main concerns of public health (1) and the major cause of disability worldwide (2). This disease causes 30 percent of all deaths in the world (3) and by 2030 this rate will increase from 17.5 in 2010 to 23 million in 2030 (4). In Iran, cardiovascular disease is the first and most common cause of death in all ages and in both genders (5). This disease causes 700 to 800 deaths (more than $39 \%$ of all deaths) in Iran each day $(6,7)$. Since cardiovascular disease involves many complications as well as high care expenditure for the patients, it has been considered as the main burden of diseases among non-communicable diseases (6); therefore,

\section{Corresponding author:}

Assistant Professor Dr. Mohabbat Mohseni, Social Determinants of Health Research Center, Institute for Futures Studies in Health, Kerman University of Medical Sciences, Kerman, Iran.

Tel: +98.3431325098, Mobile: +98.9131408140, Fax: +98.3431325094, Email: Mohabbat.Mohseni@gmail.com Received: May 15, 2017, Accepted: September 06, 2017, Published: December 2017

iThenticate screening: September 04, 2017, English editing: October 22, 2017, Quality control: December 11,2017 (C) 2017 The Authors. This is an open access article under the terms of the Creative Commons Attribution-NonCommercialNoDerivs License, which permits use and distribution in any medium, provided the original work is properly cited, the use is non-commercial and no modifications or adaptations are made. 
health organizations need a preventive population-based method to prevent this disease (1). Although cardiovascular disease is multifactorial (8), extensive research has shown that most of the deaths caused by cardiovascular diseases were due to obstruction caused by arteriosclerosis (9). However, in the population aged from 15 to 64 years, diet was ranked as the first factor of 10 health risk factors and burden of disease in Iran (10). On the other hand, the positive effect of increased intake of fruit and vegetables, and reduced consumption of salt, sugar and trans fatty acids on cardiovascular disease, has been reported in several studies (11-14). Therefore, many of these risk factors can be prevented through health education and promotion programs. Health education and health promotion models are used to change the behavior. Models form the basis for educational interventions. One of the health education and promotion models that is able to predict the effective factors on behavior change or performance is the PRECEDE model (15). This model is designed based on the principles of epidemiology, health education and health management, and is a comprehensive planning model for health promotion and life quality and includes four assessment phases: 1) Social diagnosis; 2) Epidemiological, behavioral \& environmental diagnosis; 3) Educational $\&$ ecological diagnosis; 4) Administrative \& policy diagnosis that the third phase including three main constructs (predisposing, reinforcing, and enabling), is the hallmark of this model (16). According to Green's definition, predisposing factors are those that make motivations for behavior, and include knowledge, attitude, and perceived self-efficacy. Reinforcing factors and enabling factors are those that strengthen or weaken the continuation of a behavior and make it possible for individuals to change their behavior, respectively (17). Since the PRECEDE model is a comprehensive model; it can be used in different fields (18). Various studies have shown that this model had impact on correction of eating behavior (19) and promotion of life quality (20), and is very effective compared with other models. In the previous studies performed on students, enabling factors such as access, and mothers' choice as well as facilitating factors, had correlation with consumption of healthy foods in the week before the study (21). However, due to the important role of the family, behavior of the general public could not be predicted based on the behavior of adolescents; therefore, the results obtained from the study could not be generalized to the whole of society. Also, in previous studies on predicting factors of fruit and vegetable intake, a significant and direct correlation between all the constructs of this model and also with the performance of participants, was reported, and according to the results, the main predicting factors of fruit and vegetable intake were reinforcing factors, enabling factors, and knowledge, respectively (22). In another study, predisposing factors such as belief in low-fat diet and high perceived self-efficacy in both genders, had the strongest correlation with lower fat intake (23). Inconsistencies in results and poor predictability of the previous studies have encouraged the researchers to perform the present study. The aim of this study was to determine the effective factors on eating behavior in the prevention of cardiovascular diseases and promotion of community health, based on the PRECEDE model.

\section{Material and Methods}

\subsection{Research design and sampling}

This cross-sectional study was performed in Kerman, in 2015. According to the previous studies (20, 22), approximate $\mathrm{r}$ was considered as equal to 0.2 , and Fisher scale transformation was calculated (c) equal to 200. By multiplying this number (200) in 1.5 (design effect), the sample size was estimated as equal to 350 , and considering the exclusion of $10 \%$ of participants, the final sample size was determined as 400 . In this study, participants were selected using multistage random sampling. In order to enhance the accuracy of the results, the city was divided into five regions: North, South, East, West, and Center. Two health centers were randomly selected from each region; therefore, 10 health centers were selected for the study. Then, 40 residents ( 20 men, 20 women) under the care of each health center were selected using cluster sampling method. The inclusion criteria were aged 20 to 60 years, Iranian nationality, and sufficiently literate to complete the questionnaire. The exclusion criteria included aged younger than 20 years and over sixty years, having cardiovascular diseases (according the participants record), lack of reading and writing skills, and not having permanent residence in Kerman.

\subsection{Data Collection Tools}

In this study, data were collected using a self-administrated questionnaire consisting of three parts:

\subsubsection{Part 1:}

The first part included demographic characteristics (age, gender, marital status, education, occupational status, number of family members, family history, and knowledge about their health status).

2.2.2. Part 2:

The second part was based on the constructs of the PRECEDE model and included:

1) Predisposing factors included knowledge, attitude, and perceived self-efficacy factors; knowledge consisted of 14 questions including three choice options of correct, incorrect, and do not know (the total score ranging from zero to 28), 15 questions related to attitude (such as attitude towards the effect of diet 
on cardiovascular health) and were based on a 5-point Likert scale (strongly agree, agree, neither agree nor disagree, disagree, strongly disagree), the total score ranging from zero to 30 , and 6 questions related to perceived self-efficacy (such as I can use low-sodium foods), the total score ranging from zero to 12 and were based on a 5-point Likert scale (strongly agree, agree, neither agree nor disagree, strongly disagree).

2) Reinforcing factors consisted of 6 questions based on a 5-point Likert scale (very high to very low) with the total score ranging from zero to 12 , such as "encouragement of family and friends to consume healthy foods".

3) Enabling factors consisted of 8 questions based on a 5-point Likert scale (very high to very low) with the total score ranging from zero to 16 , such as "income adequacy to buy fruit and vegetables".

2.2.3. Part 3:

The third part included 8 questions related to eating behaviors that predict cardiovascular diseases based on a 5-point Likert scale (always to never) with the total score ranging from zero to 16, such as "consumption of high-fat dairy products or high-sodium foods in the last week".

\subsection{Validity and reliability of the instrument}

The questionnaire validity was confirmed by a panel of experts (6 experts of the health department and nutrition department). To assess the content validity of the questionnaire, a pilot study was conducted prior to conducting the main project. The pilot study participants comprised of 30 subjects, who were included in the main study. Its reliability was confirmed by Cronbach's alpha and test-retest. We also estimated the internal consistency of the questionnaires (Table 1). The questionnaire was completed by the trained interviewers who went to the participants' homes.

Table 1. Result of Cronbach's alpha and intraclass correlation coefficients

\begin{tabular}{|l|l|l|l|}
\hline Structures questionnaire & Number of the questions & Cronbach's alpha & ICC \\
\hline Knowledge & 14 & 0.78 & 0.66 \\
\hline Attitude & 15 & 0.86 & 0.68 \\
\hline Perceived Self-efficacy & 6 & 0.89 & 0.79 \\
\hline Reinforcing factors & 6 & 0.76 & 0.68 \\
\hline Enabling factors & 8 & 0.88 & 0.76 \\
\hline Eating behaviors & 8 & 0.75 & 0.72 \\
\hline
\end{tabular}

\subsection{Data Analysis}

Data were analyzed using descriptive statistics (measures of central tendency, distribution, frequency, and percentage) by IBMC SPSS $\odot$ Statistics version 22 (IBMC Corp., Armonk, NY, USA) and Stata 12. For data analysis, Spearman correlation coefficient, univariate and multiple median regression were performed. By analysis of artificial neural networks, predictive power of the model constructs was determined. Given the relatively acceptable sample size $(\mathrm{n}=368)$, skewness and kurtosis parameters were used to test the normality of the data. Due to the high values of these indexes $(>1$ and $<-1)$ distribution of data was not normal; therefore, the median regression was used in STATA. The effect of demographic variables on the questionnaire constructs was investigated using univariate median regression, then using backward method and multiple median regression, the final results were obtained. Also, using a 3-layer neural network including one input layer with five neurons (knowledge, attitude, perceived self-efficacy, reinforcing factors, and enabling factors), one hidden layer, and one output layer (behavior) and also using Gaussian distribution or radial, the behavioral factors were predicted and the effect of variables on eating behaviors was determined.

\subsection{Ethics of research}

Participants were informed that participation was completely voluntary. No name was recorded on the questionnaires (No. 52923).

\section{Results}

Of 400 samples collected, 32 individuals (8\%) were excluded (due to having any of the exclusion criteria) and 368 were included in the study. The average age of participants was $35.7 \pm 11.45$ years and the mean body mass index was $25.3 \pm 5.1 \mathrm{~kg} / \mathrm{m} 2$. Most of the participants were women (54.9\%) and married (67.9\%). More than half of them (54.6\%) had a family history of diseases such as diabetes, hypertension, cardiovascular and cerebrovascular diseases. Almost half of them (49.7\%) had not undergone any check up in the past year. Also, about half of the participants $(54.3 \%)$ have academic education and the rest $(45.7 \%)$ had elementary and secondary education. The 
highest and lowest scores, mean, and standard deviation of all constructs of the questionnaire were shown in Table 2, In order to compare the constructs of the questionnaire, the scores of each scale were transformed into 0-100 scales. In this table, zero was considered as the lowest score and 100 as the highest score. Among all the constructs, knowledge (84.15 \pm 10.7$)$ had the highest and attitude, (47.84 \pm .67) the lowest average. The relationship between all variables and eating behavior was positive and significant. As shown in Table 3, perceived self-efficacy had the most, and reinforcing factors the least correlation with eating behavior. Final results about the effect of demographic variables on the questionnaire constructs using multiple median regression backward method, were shown in Table 4. As shown in Table 4, knowledge had a significant correlation with age and number of family members. The attitude and perceived self-efficacy scores of women were higher than those of men and perceived self-efficacy score of individuals who were aware of their health status was 8.33 higher than others. The relationship between enabling factors and knowledge of health status was positive and significant. In general, eating behavior and performance of the participants had a significant correlation with age, and women's eating behavior was better than that of the men. Figure 1 shows the significance of each variable (Table 5) in preventive behaviors of cardiovascular disease using artificial neural network. As shown in Table 5 and Figure 1, attitude, perceived self-efficacy, and enabling factors were respectively significant predictive factors for choosing preventive behaviors of cardiovascular diseases.

Table 2. Measures of central tendency and distribution of the questionnaire constructs

\begin{tabular}{|l|l|l|l|}
\hline Structures questionnaire & Minimum & Maximum & Mean \pm SD \\
\hline Knowledge & 39.29 & 100 & $84.15 \pm 10.7$ \\
\hline Attitude & 15 & 65 & $47.84 \pm 7.67$ \\
\hline Perceived Self-efficacy & 12.5 & 100 & $59.1 \pm 16.57$ \\
\hline Reinforcing factors & 16.67 & 100 & $60.66 \pm 14.01$ \\
\hline Enabling factors & 6.25 & 93.75 & $56.5 \pm 12.91$ \\
\hline Eating behaviors & 15.63 & 100 & $62.1 \pm 14.7$ \\
\hline
\end{tabular}

Table 3. Correlation of the PRECEDE model constructs with eating behavior using Spearman's correlation coefficient

\begin{tabular}{|c|c|c|c|c|c|c|}
\hline Variable & Knowledge & Attitude & $\begin{array}{l}\text { Perceived Self- } \\
\text { efficacy }\end{array}$ & $\begin{array}{l}\text { Reinforcing } \\
\text { factors }\end{array}$ & $\begin{array}{l}\text { Enabling } \\
\text { factors }\end{array}$ & $\begin{array}{l}\text { Eating } \\
\text { behaviors }\end{array}$ \\
\hline Knowledge & 1 & & & & & \\
\hline \multirow[t]{2}{*}{ Attitude } & $0.25^{*}$ & \multirow[t]{2}{*}{1} & & & & \\
\hline & $<0.0001^{* *}$ & & & & & \\
\hline \multirow{2}{*}{$\begin{array}{l}\text { Perceived Self- } \\
\text { efficacy }\end{array}$} & 0.2 & 0.35 & \multirow[t]{2}{*}{1} & & & \\
\hline & $<0.0001$ & $<0.0001$ & & & & \\
\hline \multirow{2}{*}{$\begin{array}{l}\text { Reinforcing } \\
\text { factors }\end{array}$} & 0.1 & 0.04 & 0.15 & \multirow[t]{2}{*}{1} & & \\
\hline & 0.63 & 0.47 & 0.004 & & & \\
\hline \multirow[t]{2}{*}{ Enabling factors } & 0.2 & 0.22 & 0.4 & 0.23 & \multirow[t]{2}{*}{1} & \\
\hline & $<0.0001$ & $<0.0001$ & $<0.0001$ & $<0.0001$ & & \\
\hline \multirow[t]{2}{*}{ Eating behaviors } & 0.21 & 0.4 & 0.45 & 0.12 & 0.31 & \multirow[t]{2}{*}{1} \\
\hline & $<0.0001$ & $<0.0001$ & $<0.0001$ & 0.024 & $<0.0001$ & \\
\hline
\end{tabular}

* The spearman correlation coefficient, ${ }^{* *}$ p-value

Table 4. The effect of demographic variables on the model constructs using multiple median regression

\begin{tabular}{|l|l|l|l|}
\hline The dependent variable & The independent variable & The regression coefficient $(0.95$ C.I.) & p-value \\
\hline \multirow{2}{*}{ Knowledge } & Age & $0.11(0.03,0.2)$ & 0.005 \\
\cline { 2 - 4 } & Family size & $0.8(0.16,1.45)$ & 0.015 \\
\hline Attitude & Sex $($ male) & $-3.3(-6.6,0.09)$ & 0.044 \\
\hline \multirow{2}{*}{ Perceived self-efficacy } & Sex (male) & $-8.33(-16.6,-0.09)$ & 0.047 \\
\cline { 2 - 4 } & Knowing your health status** & $8.33(0.17,16.5)$ & 0.045 \\
\hline Enabling factors & Knowing your health status & $6.25(0.1,12.4)$ & 0.046 \\
\hline Eating behavior & Age & $0.24(0.04,0.4)$ & 0.02 \\
\cline { 2 - 4 } & Sex (male) & $-6.97(-11.6,-2.34)$ & 0.003 \\
\hline
\end{tabular}

* Women were considered as reference and men were compared with them; ** It means medical checkups (measuring blood sugar, blood lipids, and blood pressure) in last year. 
Table 5. Significance of the questionnaire constructs in eating behavior

\begin{tabular}{|l|l|l|}
\hline Variable & Importance & Normalized percent (\%) \\
\hline Attitude & 0.324 & 100 \\
\hline Perceived self-efficacy & 0.303 & 93.5 \\
\hline Enabling factors & 0.266 & 81.9 \\
\hline knowledge & 0.093 & 28.6 \\
\hline Reinforcing factors & 0.014 & 4.3 \\
\hline
\end{tabular}

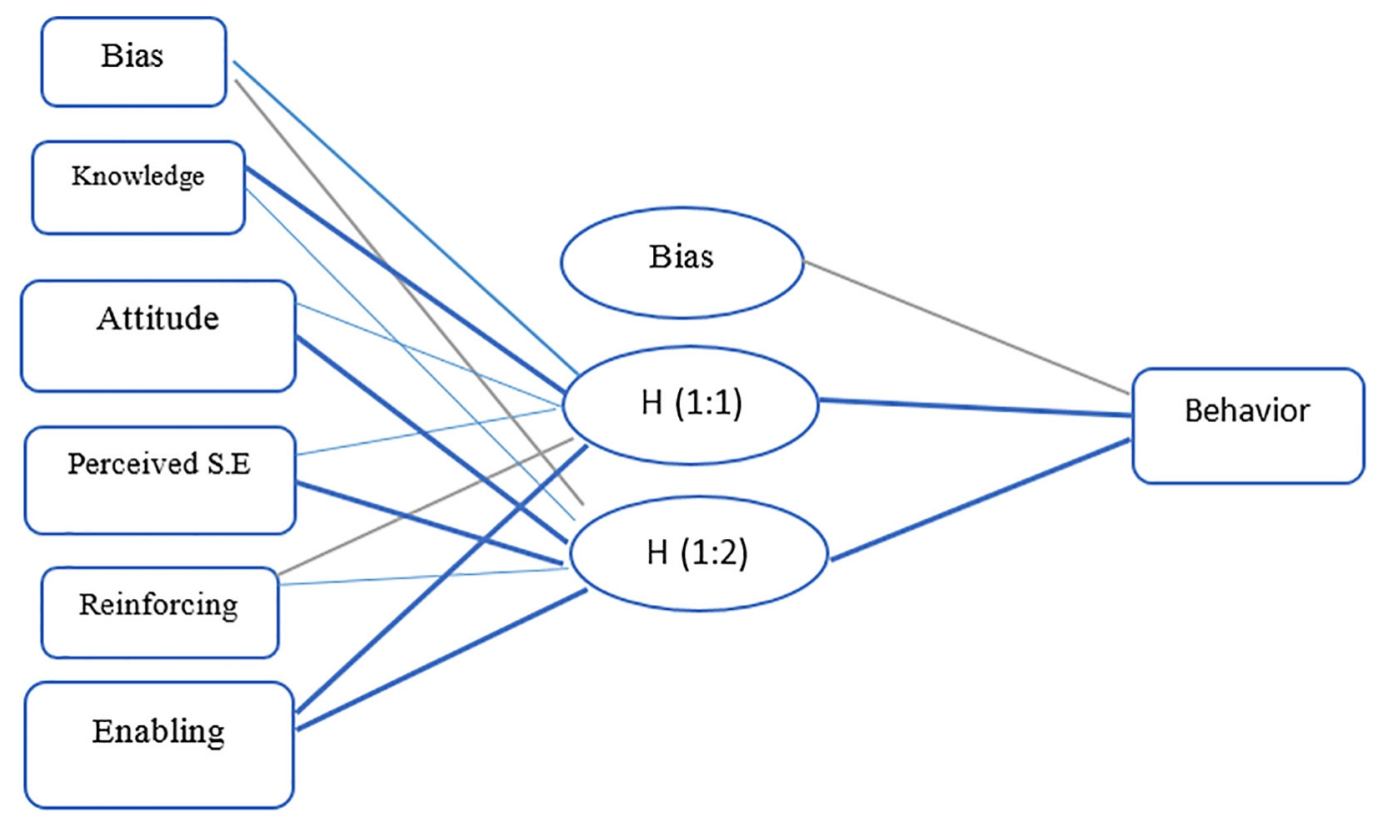

Figure 1. Significance of the questionnaire constructs in eating behavior using artificial neural network

\section{Discussion}

This was one of the first studies that assessed the effects of eating behaviors for preventing cardiovascular disease; psychosocial and environmental factors based on a theoretical framework; and determined predictive factors of eating behavior using artificial neural network. The aim of this study was to determine the effective factors of eating behaviors in the prevention of cardiovascular diseases in the community. The results showed that in both genders aged 20 to 60 years, attitude, perceived self-efficacy, and enabling factors were respectively the main predictive factors of eating behaviors in participants. It was also revealed that knowledge score of the population was good but the score of eating behavior was intermediate. Although it was revealed that information and education in this field was desirable, behavior of the population was not according to their eating knowledge, thus weak relationship between eating knowledge and behavior was reported, which was consistent with the results of similar studies (21, 23-25). Considering the average education level of the population, access to print and electronic scientific resources, media, and social networks, the knowledge level of the population was high. But analysis of population behavior showed that merely education and enhancing knowledge level of the population could not be effective in promotion or maintenance of a behavior. The results of this study showed that knowledge score of the population was desirable, which was consistent with the results of the previous studies $(22,23)$, but merely promotion of knowledge was not the main effective factor on eating behavior, and perceived self-efficacy and reinforcing factors more effectively increased healthy behaviors. Therefore, considering the gap between knowledge and performance, health providers are recommended to more effectively improve attitudes, perceived self-efficacy, and enabling factors. In this study, the low score of attitude was inconsistent with similar studies $(24,26)$. This inconsistency may be due to different demographic characteristics (such as considering both genders, different age groups, and heterogeneous participants from different regions of the city) of the population studied in this study compared with that of other studies. On the other hand, attitude was one of the main factors in predicting the eating behavior, which was consistent with the results of other studies $(24,27,28)$. Attitude is a mental and emotional entity that is affected by the family and society; therefore, by focus on the family and social environments, attitude of the individuals can be effectively affected. The results of this study showed that attitude followed by perceived self-efficacy, was the 
second predictive factor, in choosing the eating behavior; therefore, health providers are recommended to change and improve the attitude of the community. According to the results of this study, the score of perceived selfefficacy was intermediate. However, in many cross-sectional studies, perceived self-efficacy was not investigated, which was the strengths of this study compared to the similar studies. In the study of Tabasi et al. (29), the score of perceived self-efficacy was less than the average score, which was inconsistent with the results of this study. However, considering some differences in the study of Tabasi et al. (29) such as age of the participants (high school students), lack of confidence in their abilities, and dependence on parents, comparison of its results with those obtained from the present study that its participants were mostly employed and independent is not rational or precise. The results of this study showed that perceived self-efficacy was the main effective predictor of choosing eating behavior. Given that the study population were mature and had relative autonomy, score of perceived selfefficacy of them was expected to be higher than that of other age groups. Perceived self-efficacy refers to people's beliefs about their capabilities to produce designated levels of performance which can be improved by practice and organizing skills, beliefs about ability to perform skills, and management of different situations. To improve the perceived self-efficacy of the population, it is necessary to perform some interventions with an emphasis on training practical skills. Therefore, health providers are recommended to perform effective interventions to improve perceived self-efficacy of the population. According to the results, the score of reinforcing factors was intermediate, which was consistent with the Heshmati study (22). Enabling factors were significantly affected by the social environment, peers, and family, and it could be improved by group training and workshops to train family and friends. Unfortunately, the effect of the media on eating behavior was not investigated in this study, which might be one of the supposed limitations of this study. Given the important role of the media, studies on its effect will provide different results. In this study, the score of enabling factors was intermediate while in previous studies (22), was low. This inconsistency can be justified due to financial independence and income of the population in this study. Higher social conditions and economic level were the main effective factors on selection of individuals and facilitating their access to existing resources. Although the relationship between all variables and eating behavior was weak, they were significant in this study, which was inconsistent with the results of previous studies (26). In the study of Tabasi et al. (29), no statistically significant relationship between physical activity of women, predisposing factors (attitude and knowledge), or reinforcing factors was reported. This inconsistency may be due to different types of studies and population. Analysis of artificial neural network showed that attitude, perceived self-efficacy, and enabling factors were respectively the main predicting factors for eating behaviors in both genders. At the same time, the score of eating behavior had positive and significant relationship with age. The score of eating behavior in men was less than those in women. According to the obtained results, the scores of women's knowledge, attitude, perceived self-efficacy, and eating behavior were higher than those of men, and knowledge about health status was the main factor affecting the scores of perceived self-efficacy and enabling factors.

\section{The Strengths and Limitations}

This study was theory-based and performed on an acceptable population with a wide range of ages and genders. Using multiple median regression and artificial neural network based on a theoretical framework, was another strength of this study. However, there were some limitations, which included collecting data through interview. Multistage random sampling (which considered all regions of the city, the wide range of social and cultural diversities, education level, age groups, and genders) was applied, and distribution of the data was not normal. In addition, ignoring the role of social networks and media as a reinforcing factor for eating behavior was another limitation of this study.

\section{Conclusions}

According to the results of this study, knowledge level of the community was good; and self-efficacy, attitude, and enabling factors were the main predictors for eating behaviors. To prevent cardiovascular disease and enhance healthy eating behavior, it is recommended to change the attitude, and enhance self-efficacy and enabling factors in the community. We also suggest to conduct future studies assessing other effective risk factors (smoking and physical activity), and other factors affecting choosing preventive behavior. The obtained results could help experts, researchers, and health decision-makers for planning health behavior interventions.

\section{Acknowledgments:}

This article was part of the thesis submitted by the senior author for the degree of Master of Science (M.Sc.) and registered in Social Determinants of Health Research Center. Therefore, the author would like to express gratitude to Kerman University of Medical Sciences, the School of Health, the Department and Head of Health Education and Promotion, supervisors, advisors, and all people who participated in this study and helped her to perform the study. 


\section{Conflict of Interest:}

There is no conflict of interest to be declared.

\section{Authors' contributions:}

All authors contributed to this project and article equally. All authors read and approved the final manuscript.

\section{References:}

1) Seef S, Jeppsson A, Stafstrom M. What is killing? People's knowledge about coronary heart disease, attitude towards prevention and main risk reduction barriers in Ismailia, Egypt (descriptive cross-sectional study). Pan Afr Med J. doi: 10.11604/pamj.2013.15.137.1628. PMID: 24319527, PMCID: PMC3853340.

2) Sofi F, Vecchio S, Giuliani G, Marcucci R, Gori A, Fedi S, et al. Dietary habits, lifestyle and cardiovascular risk factors in a clinically healthy Italian population: the 'Florence' diet is not Mediterranean. Eur J Clin Nutr. doi: 10.1038/sj.ejcn.1602112. PMID: 15741987.

3) WHO publishes definitive atlas on global heart disease and stroke epidemic. Indian journal of medical sciences. 2004; 58(9): 405-6.

4) WHO/ISH Cardiovascular Risk Prediction Charts for WHO epidemiological sub-regions AFR D and AFR E. Prevention of Cardiovascular Disease Pocket Guidelines for Assessment and Management of Cardiovascular Risk. 2007.

5) Mohsen N. Change the face of health in iran. Iranian Journal of Epidemiology. 1385; 1(3).

6) Who J, Consultation FAO Expert. Diet, nutrition and the prevention of chronic diseases. World Health Organ Tech Rep Ser. 2003.

7) Finegold JA, Asaria P, Francis DP. Mortality from ischaemic heart disease by country, region, and age: statistics from World Health Organisation and United Nations. Int J Cardiol. 2013; 168(2): 934-45. doi: 10.1016/j.ijcard.2012.10.046. PMID: 23218570, PMCID: PMC3819990.

8) Park JE. Text book of prevative and sodial medicen atreatise on community health .17th ed. 2002.

9) McGill HC Jr, McMahan CA, Gidding SS. Preventing heart disease in the 21st century: implications of the Pathobiological Determinants of Atherosclerosis in Youth (PDAY) study. Circulation. doi: 10.1161/CIRCULATIONAHA.107.717033. PMID: 18316498.

10) Sabzmakan L, mazloomy MS, Morowatisharifabad MA, Mohammadi E, Naseri MH, et al. Patients, Experiences with Cardiovascular Disease Risk Factors and Healthcare Providers of Determinants of the Feeding Behavior: A Qualitative Directed Content Analysis. Iranian Journal of Endocrinology and Metabolism. 2013; 15(3): 292-302.

11) Finks SW, Airee A, Chow SL, Macaulay TE, Moranville MP, Rogers KC, et al. Key articles of dietary interventions that influence cardiovascular mortality. Pharmacotherapy. 2012; 32(4): e54-87. doi: 10.1002/j.1875-9114.2011.01087.x.

12) Mason H, Shoaibi A, Ghandour R, O'Flaherty M, Capewell S, Khatib R, et al. A cost effectiveness analysis of salt reduction policies to reduce coronary heart disease in four Eastern Mediterranean countries. PLoS One. 2014; 9(1): e84445. doi: 10.1371/journal.pone.0084445. PMID: 24409297, PMCID: PMC3883693.

13) Guallar-Castillon P, Rodriguez-Artalejo F, Lopez-Garcia E, Leon-Munoz LM, Amiano P, Ardanaz E, et al. Consumption of fried foods and risk of coronary heart disease: Spanish cohort of the European Prospective Investigation into Cancer and Nutrition study. BMJ. 2012; 344: e363. doi: 10.1136/bmj.e363. PMID: 22275385, PMCID: PMC3265571.

14) Schneider S, Diehl K, Bock C, Herr RM, Mayer M, Gorig T. Modifying health behavior to prevent cardiovascular diseases: a nationwide survey among German primary care physicians. Int J Environ Res Public Health. 2014; 11(4): 4218-32. doi: 10.3390/ijerph110404218. PMID: 24739770, PMCID: PMC4025039.

15) Salehi L. Application of Programmable asked to improve feeding behaviors related to cardiovascular disease in a rural community. Iranian Journal of Epidemiology. 2010; 6.

16) Mohsen S. Theories and models and methods of health education and health promotion. Tehran: Sobhan; 1391.

17) Green LW, Kreuter MW. Health Promotion Planning: An Educational and Ecological Approach with Power Web Bind-In Card. McGraw-Hill; 2004.

18) Afkari ME, Solhi M, Matin H, Hoseini F, Mansoorian M. The efficiency of educational intervention based on PRECEDE educational method in the promotion of life quality of the aged under the coverage of Tehran Cultural House of Aged People 2009. Iranian Journal of Ageing. 2011; 5(4). 
19) Colodny N, Miller L, Faralli M. The development of a feeding, swallowing and oral care program using the PRECEDE-PROCEED model in an orphanage-hospital in Guatemala. Int J Speech Lang Pathol. 2015; 17(2): 127-37. doi: 10.3109/17549507.2014.927924. PMID: 25014491.

20) Baghianimoghadam MH, Mohamadi S, Baghianimoghadam M, Falahi A, HS R. Survey on Quality Of Life related factors in patients with Peptic Ulcer based on PRECEDE Model in Yazd, Iran. Journal of Medicine and Life. 2011; 4(4).

21) Dabone C, Delisle H, Receveur O. Predisposing, facilitating and reinforcing factors of healthy and unhealthy food consumption in schoolchildren: a study in Ouagadougou, Burkina Faso. Glob Health Promot. 2013; 20(1): 68-77. doi: 10.1177/1757975913476905. PMID: 23563781.

22) Heshmati H, Behnampour N, Homaei E, Khajavi S. Predictors of fruit and vegetable consumption among female high school students based on PRECEDE model. Iranian Journal of Health Education and Health Promotion. 2014; 1(4): 5-14.

23) Watters JL, Satia JA. Psychosocial correlates of dietary fat intake in African-American adults: a crosssectional study. Nutr J. 2009; 8: 15. doi: 10.1186/1475-2891-8-15. PMID: 19320975, PMCID: PMC2667442.

24) Nowbar AN, Howard JP, Finegold JA, Asaria P, Francis DP. 2014 global geographic analysis of mortality from ischaemic heart disease by country, age and income: statistics from World Health Organisation and United Nations. Int J Cardiol. 2014; 174(2): 293-8. doi: 10.1016/j.ijcard.2014.04.096. PMID: 24794549, PMCID: PMC4045191.

25) Noroozi E, Miri MR, Gol Mohammadi S. Application of Behavioral Analysis phase of PRECEDE Model for Quality of Life Survey in Postmenopausal women in Birjand. Journal of Birjand University of Medical Sciences. 2012; 19(3): 312-23.

26) Falahi A, Nadrian H, Mohammadi S, Baghiyani Moghadam M. Utilizing the PRECEDE Model to predict quality of life related factors in patients with Ulcer Peptic Disease in Sanandaj, Kurdistan, Iran. Payavard Salamat. 2009; 3(2): 30-43.

27) Norozi E, Mostafavi F, Hasanzadeh A, Moodi M, Sharifirad G. Factors affecting quality of life in postmenopausal women, Isfahan, 2011. Journal of education and health promotion. 2012;2: 58.

28) Nadrian H, Morowatisharifabad MA, Bahmanpour K. Development of a Rheumatoid Arthritis Education Program using the PRECEDE_PROCEED model. Health Promotion. 2011; 1(2): 118-29.

29) Tabasinejad N, Ahmadi Tabatabaei SV, Khanjani N, Mohseni M. English Title: Predicting of Factors influencing physical activity in women for the prevention of osteoporosis according the Precede Model. Health-Based Research. 2015; 1(2): 155-66. 